\title{
Research on the Xishu Garden Based on Landscape Identity
}

\author{
Dan Wu \\ Chengdu Art Vocational College, Chengdu, Sichuan, 611430, China
}

Keywords: Xishu Garden, Landscape identity, West Sichuan towns

\begin{abstract}
Xishu Garden is a regional garden located in Sichuan, China, which is an integral part of Chinese garden system. Landscape identity is a problem about the definition and recognition of Xishu Garden's regional style. This paper studied the connotation of landscape identity, Xishu Garden, West Sichuan towns, western Sichuan customs and so on under the landscape scale vision to make a preliminary analysis of the research ideas of Xishu Garden landscape identity.
\end{abstract}

\section{Introduction}

Sichuan's Humanistic Historical process is unique and complex. Xishu Garden's landscape identification should be regarded as a subject of environmental history. In 1450, Alberti, the first western garden theorist, regarded the relationship between environment and house, which is the relationship between environment and the viewer in it, as the basis of landscape recognition. Therefore, the study of Xishu Garden should be based on the whole human settlement space system, such as Sichuan's natural geography, ecological stage and immigrants from Sichuan, that is, the great vision of Sichuan's urban history.

\section{The Connotation of Landscape Identity}

In Chinese, as to the word scenery, most people tend to appreciate the meaning of leisure. The purpose of this paper is to emphasize the significance of landscape as a social, economic, political and cultural carrier. Like the garden culture of ancient and modern times, the visual reproduction and semantic reproduction of the landscape will make the silent landscape image spread, sound and express the landscape identity of a place. For example, European landscape paintings and Chinese landscape paintings, British lakeside poets chant and Chinese landscape pastoral poetry. Sichuan landscape and Xishu Garden are not lack of such performance, and the architectural couplets are examples. Scenery and its semantic expression of scenic intention are unique and unparalleled in the world landscape culture. This form of cultural valuation has become the aesthetic permanent product of local scenery and landscape, as well as the concrete embodiment of landscape identity.

\section{Xishu Garden in View of Landscape Scale}

In the eyes of geologists, Sichuan is unique and complex in China, and the biggest cultural difference in China. Judging from this large scale, Sichuan is near the Qinghai-Tibet Plateau in the west, in the east according to the danger of the three Gorges of the Yangtze River, in the north in the Qinling Mountains and in the Bashan, and in the south in the barrier of the Yunnan-Guizhou Plateau. Sichuan is near the Qinghai-Tibet Plateau in the west, the three Gorges in the east, the Bashan Mountain in the north, and the Yunnan-Guizhou Plateau in the south. Within the province, there is a great difference in height, less than 500 meters above the elevation of the plains, hills, valleys accounted for 18, and the rest are above 500 meters above sea level. Gan, A, Liang three highlands and quasi-plateau ethnic minority autonomous prefectures cover an area of 300000 square kilometers. The Chengdu Plain in the western heart of Sichuan Province is protected by this scale of terrain. Xishu Garden's landscape identity is a understanding of Sichuan people with their own physical strength, mental power, who travelled throughout Sichuan and southwest of the mountains and rivers in the course of thousands of years of history. The regional landscape in the west of 
Sichuan is an all - in - one. The landscape impression of Sichuan on the large scale is noble and magnificent, and it is a wild scenery beyond people's imagination. In western Sichuan, small-scale towns, pastures and gardens, people see the beautiful landscape of the land of abundance. In China, Sichuan is unique in the contrast and fusion of large-scale grandeur and small-scale Juan-xiu. And the reason for this uniqueness is precisely the role of the unique geological bottom structure in Sichuan, which determines the unique geomorphological characteristics of the region, and supports the unique ecosystem of the region. It also determines the survival and settlement mode of immigrants to Sichuan and the aesthetic value of cultural people to Sichuan's natural and humanistic landscape.

\section{The Local Color of Xishu Garden in Western Sichuan}

In this paper, Xishu Garden is limited to various types of garden individuals whose scale is relatively small. This kind of garden itself is an organic component of the space texture of the market space of its town or country settlement, and its internal spatial pattern and interface. Surrounded by the organic integration of the town in the space texture structure. Therefore, western Sichuan towns and rural settlements are the local color of Xishu Garden.

Since 1982, there has been 7 cities recognized as a national historical and cultural city in Sichuan Province - Chengdu, Zigong, Yibin, Luzhou, Dujiangyan, Leshan and Langzhong. The number of provincial historical and cultural cities is 25, and the number of provincial historical towns is 21. The relevant departments of Sichuan province have studied the famous villages of ancient towns, and obtained quite gratifying results, which provides systematic information for the history and present situation of the local color of Xishu Garden. Generally speaking, the following three aspects of the relevant understanding can be obtained from the results of these studies.

\subsection{Planning characteristics of ancient towns in western Sichuan}

More than 95\% of the ancient towns in western Sichuan are located along rivers. They are convenient for transportation, and are easy to build and easy to collect water. The terrain of Sichuan is complex, but the natural environment is used to shape the characteristics of towns in the construction of ancient towns. For example, Guanxian ancient city was built because of water conservancy with half mountain and half city, also, mountain and water forest city Weir merges into one body. Langzhong is the circulation on three sides of Jialing River. Jinping Mountain across the river, so that the city has everywhere Xuan window towards the view of Jinping effect. The master plan and plane layout of many ancient towns in western Sichuan are informal and flexible: boat Street, Triangle Street, trapezoidal Street, Longzhuang Street, each with its own characteristics. The ancient town's buildings and streets are pleasant in scale, and the living atmosphere is cordial and warm, thus forming a vivid impression of the town landscape: in the large area of green tile powder wall houses, the towering city towers are towering, temples, Yazu, bell towers, and drum towers are scattered between them. Theatrical buildings, archways, or streets, or at the end of the road, open and closed, set off against reality. The wall of houses on both sides of the street is constantly changing, overlooking the geomantic pagoda outside the town, either for the opposite view, or for the landmark, or for the combination of the artificial environment and the natural environment of the town. This picture can be seen in woodcut illustrations of historical landscape information.

\subsection{The characteristics of urban architecture in western Sichuan}

As an important landscape element, architecture is an important point to understand the regional characteristics of Xishu Garden. Although Sichuan ancient architecture has the commonness of Chinese ancient architecture in general-wooden frame, brick and tile lifting large roof, but it has its regional characteristics. (1) Cultural characteristics of Sichuan people. Sichuan people has a distinctive immigrant color, so the ancient architecture of western Sichuan takes ancient Shu culture as its root and takes it as an integral part. In different historical periods, it receives all kinds of external humanistic factors. (2) Measures tailored to local conditions. Western Sichuan ancient architecture showed a strong sense of environmental awareness, landscape awareness, and it set off 
by the environment, environment as the background, the building into the environment. Although the combination of architectural groups still adheres to the central axis rule, taking the quadrangle as the basic technique, it strives to comply with the environment. The building is layered by height difference, arranged on the platform of all levels, forming layer by layer body potential, and according to the axis of topographic turning, adopting the short passage and guiding treatment, the building space on the three-dimensional undulating terrain will be formed as a whole, and the hanging foot floor will be adopted. Flat platform to expand the building space, so that its layout is free, flexible and natural. The streets of Xiaochang Town are arranged along the river and bend according to the water potential, and according to the vertical height difference of the contour line, forming the tendency of continuous overlapping and falling. The streets are connected with each other with stone level, and the space of the streets is changeable. West Sichuan is rainy and less sunshine, so the roof often has a sharp slope, deep eaves, street colonnade width up to 3 meters. Roof lift fold, obvious anti-space, both rain-proof drainage and lighting effect. The cornice is mostly high and low before and after, preventing the back eaves from dripping with rain and wet walls. (3) Obtaining raw material locally. There are many types of wood, such as bamboo wall, plank wall (board wall), adobe wall, earth wall, Sanhe wall, stone wall and so on. Roof uses small green tile, gray tube tile, and the important buildings also uses glazed tiles, other such as earth roof, grass roof, plank roof, bark roof, all based on local materials with strong local atmosphere. The ancient architecture of western Sichuan has the commonness of the Chinese classical wooden frame architecture, which mostly adopts the piercing bucket structure, and the plane layout is based on the standard of "interframe" and is highly standardized. Because of the rich production of good wood (pine, fir, south, camphor), it is not lack of exquisite workmanship. As for large-space palatial buildings, the mixed structure of lifting beams and piercing buckets is used, and Sichuan bucket arch is more inclined arch, which has its own characteristics. As for large-space palatial buildings, the mixed structure of lifting beams and piercing buckets is used, and Sichuan bucket arch is more inclined arch, which has its own characteristics.

\subsection{West Sichuan garden}

Garden is a very delicate architectural space in the town, as an important architectural complex expressed by the aesthetics of the urban landscape. Each place has its streams in from all over the country, and the cultural geography connotation of western Sichuan garden is also quite rich. As a cultivated land of immigrants in different historical periods, it has formed a kind of garden variety that is especially developed in Sichuan-Tongxiang Pavilion and Guild Hall. In different periods, Sichuan literati, Xianda people built, or later generations to commemorate its track achievements of other courts, ancestral halls, is also an introduction of attention to the garden species. According to historical texts and modern archaeological excavations, the earliest gardening activities in Sichuan began in the 4th century B.C., after Qin Dynasty destroyed Shu, and it began in Chengdu. In fact, the names used in western Sichuan cities so far are quite ancient, such as Chengdu, Pixian, Guanghan and so on, all of which have been used since the beginning of the founding of the cities in the Qin, Han and Tang dynasties. Xishu Garden began in Chengdu. Chengdu is built in accordance with the two rivers near Funan River. In order to get the benefit of the two rivers facing the wind, the axis is not fixed according to the geographical longitude and latitude, but the vertical axis of 30 degrees by the west of the south is basically arranged. This pattern of urban axis layout followed the meridian as the vertical axis to layout the building until the Ming Dynasty. So far in

Chengdu, these two axes have been used in a compatible way. This is the Chengdu garden with the local background color. In the history of more than 2300 years, it became the capital of the local feudal government seven times, and the capital of the peasant uprising regime twice. Its city name doid not change, the city site had not moved. There are many ancient garden sites in the towns around Chengdu. For example, the private garden of the old house of Wang Zhuo, king of Linqiong in the western Han dynasty. King Zhuo was the aristocrat of Jing Chu who entered Sichuan, so it brought the influence of Chu style to Sichuan. People build gardens by natural landscape, and temples, gardens, such as Emei Mountain Temple, Qingcheng Mountain Tianshi Cave, Leshan 
Wuyou Temple and so on.

\section{Xishu Garden's Background Color in Western Sichuan}

The western Sichuan cities and towns are living and consuming, and people can enjoy the leisurely life regardless of their poverty. As descendants of immigrants, they are clearly visible inclusive, not exclusive, not conservative. Sichuan people are rich in sensibility, curiosity, good at expression, sensitive to information, also, they love to say, want to say. In today's reality of the various Xishu Garden, you can see the scene of Sichuan people, for the western Sichuan style and customs, it seems that can be summed up in the following paragraphs: (1) Bashu is very gentle and elegant. The integration of three foreign cultures and ancient Shu civilization is neither influenced by foreign culture nor bound by orthodox thought. The fusion of three foreign cultures, Jingchu, Qinlong and Central Plains with the ancient Shu civilization is neither influenced by foreign culture nor bound by orthodox ideas. (2) They enjoy heaven's paradise, leisurely and quiet. (3) They like drinking tea in Sichuan. Sichuan garden tea house quantity is numerous, also it is the tea industry foundation. (4) Food in Sichuan, taste in Chengdu. Xishu Garden, a collection of north and south cuisines from east to west, also enjoys eating in gardens. (5) Sichuan people enjoy fun and gardens are loved by themselves. Some people wrote about Chengdu people's good travel in Yuan Dynasty, and the various commercial cultures and festivals are also rich and colorful. This is the foundation of Xishu Garden's life, the basis of understanding the gardening machine and the orientation of its function, the reason of its existence and the internal cause of its vitality.

\section{Summary}

The research on Xishu Garden is a branch of Chinese classical garden system. The purpose of this paper is to make a preliminary analysis for the ideas of Xishu Garden landscape identity.

\section{References}

[1] Yan Wenli, Wu Linjia, Guo Li. Study on Landscape Art of Western Shu Landscape[J]. Anhui Agricultural Science, 2018, 46(15): 92-94.

[2] Sun Dajiang, Yang Yupei, Tang Qin, Chen Qibing. Recalling Mr. Wang Shaozeng and exploring the Western Shu Gardens[J]. Chinese garden,2018,34(02): 70-73.

[3] Jiang Tao, Li Junzhuo, Liu Lanfang, Sun Dajiang, Chen Qibing. A preliminary study on artistic Conception Construction of Bamboo Landscape in Western Shu Garden[J]. Shandong Forestry Science and Technology, 2016, 46(04): 104-106.

[4] Jiang Tao, Li Chunong, Chen Qibing, Yu Xiaofang. A study on the characteristics of the Landscape name of the Celebrity Memorial Gardens in Western Shu [J]. Guangdong Garden: 2015, 37(02): 65-67. 